\title{
Molecular Identification and Antimicrobial Potential of Streptomyces Species from Nepalese Soil
}

\author{
Karan Khadayat, ${ }^{1,2}$ Dawa Dindu Sherpa, ${ }^{2}$ Krishna Prakash Malla, ${ }^{2}$ Sunil Shrestha, ${ }^{2}$ \\ Nabin Rana, ${ }^{2}$ Bishnu P. Marasini, ${ }^{2}$ Santosh Khanal, ${ }^{3}$ Binod Rayamajhee, ${ }^{3}$ \\ Bibek Raj Bhattarai, ${ }^{1}$ and Niranjan Parajuli $\mathbb{1}^{1}$ \\ ${ }^{1}$ Central Department of Chemistry, Tribhuvan University, Kirtipur, Kathmandu, Nepal \\ ${ }^{2}$ Department of Biotechnology, National College, Tribhuvan University, Naya Bazar, Kathmandu, Nepal \\ ${ }^{3}$ Department of Microbiology, National College, Tribhuvan University, Naya Bazar, Kathmandu, Nepal \\ Correspondence should be addressed to Niranjan Parajuli; nparajuli@cdctu.edu.np
}

Received 4 July 2020; Accepted 12 August 2020; Published 27 August 2020

Academic Editor: Sujata Prasad

Copyright (c) 2020 Karan Khadayat et al. This is an open access article distributed under the Creative Commons Attribution License, which permits unrestricted use, distribution, and reproduction in any medium, provided the original work is properly cited.

\begin{abstract}
Streptomyces are widely used for the production of secondary metabolites with diverse biological activities, including antibiotics. The necessity of alternative antimicrobial agents against multidrug-resistant pathogens is indispensable. However, the production of new therapeutics is delayed in recent days. Thus, the isolation of new Streptomyces species has drawn attention. Nepal-a country rich in biodiversity-has got high possibilities for the discovery of members of actinomycetes, especially in the higher altitudes. However, in vain, only a few screening research works have been reported from Nepal to date. Streptomyces species were isolated on ISP4 media, and characterization was performed according to morphological similarity and 16S rRNA sequence similarity using bioinformatic tools. Ethyl acetate extracts of Streptomyces species were prepared, and the antimicrobial activity was carried out using agar well diffusion technique. In this report, 18 Streptomyces species isolated from the soil were reported based on sequence analysis of $16 \mathrm{~S}$ rRNA. Among them, 12 isolates have shown antibacterial activity against extended-spectrum beta-lactamase- (ESBL-) producing Escherichia coli. Here, we have also analyzed 16S rRNA in 27 Streptomyces species whose whole-genome sequence is available, which has revealed that some species have multiple copies of the $16 \mathrm{~S}$ gene $(\sim 1.5 \mathrm{~kb})$ with significant variation in nucleotides. In contrast, some Streptomyces species shared identical DNA sequences in multiple copies of $16 \mathrm{~S}$ rRNA. The sequencing of numerous copies of $16 \mathrm{~S}$ rRNA is not necessary, and the molecular sequencing of this region is not sufficient for the identification of bacterial species. The Streptomyces species-derived ethyl acetate extracts from Nepalese soil demonstrate potential activity against ESBL-producing E. coli. Thus, they are potential candidates for antibiotics manufacturing in the future.
\end{abstract}

\section{Background}

Multidrug-resistant pathogens have drawn global attention as a significant challenge to treat and prevent the growing number of infectious diseases $[1,2]$. Such resistance typically occurs as a result of drug inactivation, target alteration, and reduced accumulation unsettled to decreased permeability and/or increased efflux [3]. Novel organisms with the capacity to produce new secondary metabolites or therapeutic agents are immediately required to combat the fatal diseases caused by such antibiotic-resistant pathogens [4, 5].
Researchers have continuously explored novel, sustainable, potent, and broad-spectrum bioactive compounds from diverse sources, including Streptomyces species [6]. Streptomyces, the largest genus of class actinobacteria, is a Grampositive, spore-forming, filamentous, and aerobic bacterium [7]. Streptomyces genome contains more than 20 gene clusters to secondary metabolites of higher clinical importance, including antibiotics that could tackle the rise of antimicrobial resistance [8]. About two-thirds of natural antibiotics are isolated from actinomycetes, of which $70 \%$ is produced from Streptomyces and accounts for $75 \%$ of 
clinically available antibiotics used in the treatment of multiple infections in humans [9-12]. Many studies report that Streptomyces have tremendous potential to yield secondary metabolites, including anticancer drugs, antibiotics, growth factors, and herbicides [13]. The higher percentage of GC content $(\sim 70 \%)$ is present in Streptomyces spp. The $16 \mathrm{~S}$ rDNA analyses and DNA-DNA hybridization are significant distinguishing properties that separate Streptomyces from other actinobacteria. Streptomyces from extreme or untouched habitats such as high altitude $[14,15]$ and desert $[16,17]$ are isolated and cultured to discover novel antibiotics. Extended-spectrum beta-lactamases (ESBLs) produced primarily by the bacteria Escherichia coli and Klebsiella pneumoniae confer resistance to most beta-lactam antibiotics, including penicillins and cephalosporins. Infections caused by these bacteria are being difficult to treat globally, and the mortality rate has sharply increased along with prolonged hospital stay and greater economic burden [18].

Due to the unique geographical niche of Nepal, soil microbes, such as Streptomyces, have a high probability of producing novel secondary metabolites of diverse clinical value, which is much anticipated in the health care sector. In this report, soil samples from 14 different environments of Nepal with varying from $86 \mathrm{~m}$ to $4,026 \mathrm{~m}$ above the sea level in altitude were collected for the isolation of Streptomyces species with antibacterial properties. This study is mainly aimed at the screening of Streptomyces species that can produce metabolites for the inhibition of ESBL-producing clinical isolate of $E$. coli, and their molecular identification using 16S rRNA sequencing. Besides, 16S rRNA found in other Streptomyces species reported in GenBank is also reviewed for further scientific discourse.

\section{Methods}

2.1. Collection and Pretreatment of Soil Sample. The soil samples were collected from 14 different habitats of Nepal (altitude from $86 \mathrm{~m}$ to $4,026 \mathrm{~m}$ above the sea level) such as gardens, cultivated fields, barren land, open fields, organic manure pits, and soil from conserved forests, as shown in Supplementary Materials (Table S1). The samples were collected digging around 5-10 $\mathrm{cm}$ depth from the surface of the earth. Soil samples were then packed in sterile polythene bags, labeled, and brought to the laboratory. They were airdried for 3-4hrs at $45^{\circ} \mathrm{C}$, crushed, and sieved before use for the isolation of Streptomyces species [19].

2.2. Isolation of Streptomyces. One gram of soil was dissolved in $10 \mathrm{~mL}$ of sterile distilled water in the test tube and vortexed vigorously for 5 minutes. Then, the suspension was heated at $80^{\circ} \mathrm{C}$ for 30 minutes and serially diluted up to $10^{-4}$ in the laminar hood; then $100 \mu \mathrm{L}$ of each dilution was placed on the International Streptomyces Project 4 (ISP4) agar medium supplemented with nalidixic acid $(20 \mathrm{mg} / \mathrm{mL})$ and cycloheximide $(50 \mathrm{mg} / \mathrm{mL})$ (ISP4: $10 \mathrm{gm}$ starch, $1 \mathrm{gm}$ dipotassium phosphate $\left(\mathrm{K}_{2} \mathrm{HPO}_{4}\right), 1$ gm calcium carbonate, $1 \mathrm{mg}$ ferrous sulfate, $1 \mathrm{mg}$ manganese chloride, $1 \mathrm{mg}$ zinc sulfate, $18 \mathrm{gm}$ Bacto agar, $1 \mathrm{gm}$ sodium chloride, $2 \mathrm{gm}$ ammonium sulfate, and $1000 \mathrm{~mL}$ distilled water at $\mathrm{pH}$ $7.0 \pm 0.1$ ). The inoculum was then appropriately spread using a sterile glass spreader until the plates were dry and incubated at $28^{\circ} \mathrm{C}$ for $7-12$ days [20].

2.3. Characterization of Streptomyces. The characterization of Streptomyces was performed based on their Gram staining, growth pattern, colony morphology, and the formation of soluble pigments, as suggested by Bergey's Manual of Systematic Bacteriology, Second Edition, Vol. 5, The Actinobacteria, Part A [21]. All of the isolates were grown on ISP4 media, and the morphology of each isolate was visually observed (such as colony characteristics, an earthy odor, spore formation, and aerial and substrate mycelia of colonies). Sugar utilization tests and physiological tests such as motility, salt tolerance, and temperature tolerance were also performed.

2.4. Genomic DNA Extraction and 16S rRNA Amplification of Isolated Streptomyces Strains. Phenotypically identified isolates of Streptomyces were cultured on trypticase soy broth (TSB) using glass beads in $100 \mathrm{ml}$ conical flasks for 3 days at $28^{\circ} \mathrm{C}$. After incubation, the supernatant part was discarded, and mycelia were harvested from the broth by centrifugation at 4,000 rpm for $15 \mathrm{~min}$. Genomic DNA was isolated by the standard phenol-chloroform method from the harvested mycelia [22]. The universal primers $27 \mathrm{~F}: 5^{\prime}$-AGAGTTTGATCMTGGCTCAG-3' and 1492 R:5'-ACGGYTACCTTGTTACGACTT- $3^{\prime}$ were used for amplification of $16 \mathrm{~S}$ rRNA from genomic DNA. The amplification was carried out in a $50 \mu \mathrm{L}$ of total volume by using $125 \mathrm{ng}$ of genomic DNA as a template with $2 \mathrm{X}$ premix (Taq polymerase) and $10 \mu \mathrm{M}$ of each primer. PCR conditions were maintained as follows: initial $5 \mathrm{~min}$ for denaturation at $95^{\circ} \mathrm{C}$, followed by 35 cycles of $30 \mathrm{~s}$ at $95^{\circ} \mathrm{C}, 30 \mathrm{~s}$ at $51.4^{\circ} \mathrm{C}$, and $120 \mathrm{~s}$ at $72^{\circ} \mathrm{C}$, and a final extension of $10 \mathrm{~min}$ at $72^{\circ} \mathrm{C}$ (TAKARA Thermal Cycler, Japan). The amplified products were examined by $0.8 \%$ agarose gel electrophoresis [23] stained by ethidium bromide. The purified PCR products were sequenced using the same primers, $27 \mathrm{~F}$ and 1492R (Macrogen, Inc., South Korea).

2.5. Sequence Analysis. The homology search of partial DNA sequences of $16 \mathrm{~S}$ rRNA was performed by comparing them with the public database (NCBI) using the standard basic local alignment search tool (BLAST) program. Multiple alignments were conducted using Clustal [24], and the phylogenetic tree was made using MEGA version 6.0 by the neighbor-joining method with bootstrap values calculated from 1,000 replications (Supplementary Materials, Figure S1) [25]. On the other hand, the multiple copies of $16 \mathrm{~S}$ rRNA present in the assembled genome sequencing of the other 27 Streptomyces species given in the website (JGI IMG Integrated Microbial Genomes and Microbiomes, 2019) was evaluated through multiple DNA-DNA sequence alignments using Clustal W (Supplementary Materials, Table S3). 
2.6. Extraction of Bioactive Compounds. The isolated Streptomyces species were first cultured in the TSB and incubated at $30^{\circ} \mathrm{C}$ for three days for the preparation of inoculum. After full growth, 1\% Streptomyces mycelium was transferred aseptically into the fresh TSB and again incubated at $30^{\circ} \mathrm{C}$ for seven days. An equal volume of organic solvent, ethyl acetate, was used for extracting the bioactive compounds to an equal volume of culture. The mixture of culture with an organic solvent was shown in two layers (organic layer contained secondary metabolites) and incubated overnight in a rotary shaker. The mixture was then centrifuged, and the supernatant was taken. The concentrated supernatant was further used for antimicrobial activity.

2.7. Antimicrobial Activity against ESBL-Producing E. coli. The ESBL-producing clinical isolate of E. coli was already confirmed by coauthors in the previous study [26]. Mueller-Hinton agar (MHA) plates were spread with test organisms (0.5 McFarland turbidity standard) using a sterile cotton swab. MHA plates were bored with $6 \mathrm{~mm}$-diameter sterile cork borer, and $30 \mu \mathrm{L}$ ethyl acetate extract was loaded into wells. The plates were then incubated at $37^{\circ} \mathrm{C}$ for $24 \mathrm{~h}$, and the zone of inhibition was measured [27]. To validate the experiment, negative control (ethyl acetate) and positive control ( $1 \mathrm{mg} / \mathrm{mL}$ neomycin) were also maintained, and each experiment was triplicated. We had also examined the antimicrobial activity of some isolates with Staphylococcus aureus (ATCC 25923), E. coli (ATCC 25922), and K. pneumoniae (ATCC 700603) by the perpendicular streaking method as primary screening.

\section{Results}

3.1. Characterization of Streptomyces. Soil samples collected from various environments varied in texture, and 18 Streptomyces isolates were successfully isolated (Table S1). Among them, no isolate was isolated from the soil of Rasuwa, two isolates from Thali, Dhangadi, Chitlang, Tapoban, and Taudaha soil and one isolate from each remaining eight types of soil. The isolates were identified as Streptomyces based on their mycelial and cellular morphology observed under a microscope and no other species such as Nocardia spp. and Micromonospora spp. were obtained. All these isolates attained maximum growth after seven days of incubation with colored sporulation ranging from dark grey, grey, dark brown, brownish, whitish, and yellowish-white, as indicated in Figure 1. The microscopic examination of actinomycete strains showed Gram-positive with hair-like mycelium, as shown in Figure 2. The physiological test showed that the isolates were nonmotile, unable to produce hydrogen sulfide, optimum growth between $28^{\circ} \mathrm{C}$ and $37^{\circ} \mathrm{C}$, and able to tolerate up to $5 \% \mathrm{NaCl}$. In contrast, the sugar utilization test revealed that most of the strains under study were able to utilize glucose, arabinose, sucrose, xylose, and fructose but were not able to utilize mannitol, inositol, raffinose, and rhamnose.
3.2. Antibacterial Assays. The antibacterial activity of ethyl acetate extract $(50 \mathrm{mg} / \mathrm{mL})$ of isolates was evaluated against ESBL-producing clinical isolates of $E$. coli using well diffusion method. The results showed that crude extract exhibited a zone of inhibition ranging from 10 to $13 \mathrm{~mm}$ (size of wells: $6 \mathrm{~mm}$ ) against ESBL-producing E. coli as compared to positive control neomycin with $19 \mathrm{~mm}$ zone of inhibition, as shown in Figure 3 and Supplementary Materials, Table S2. Some pure isolates also showed antimicrobial activity against S. aureus (ATCC 25923), E. coli (ATCC 25922), K. pneumoniae (ATCC 700603), and Salmonella typhimurium (ATCC 14028) (data not included in this report).

3.3. 16S rRNA Amplification. The PCR amplification of $16 \mathrm{~S}$ rRNA using a set of universal primers $27 \mathrm{~F}$ and $1492 \mathrm{R}$ resulted in $\sim 1.5 \mathrm{~kb}$ product as compared to $1 \mathrm{~kb}$ ladder (New England Biolabs) for all isolates. The amplified products were then purified with DNA Clean and Concentrator ${ }^{\mathrm{TM}}-5$ (Catalog no. D4003), Zymo Research (USA), following the manufacturer's instructions, and the DNA sequencing using universal primers was performed in Macrogen Inc., South Korea.

3.4. Molecular Characterization. The proximity of the $16 \mathrm{~S}$ rRNA gene of the isolates was compared with the sequences available in public databases (GenBank, EMBL, and DDBJ) using the Nucleotide Basic Local Alignment Search Tool-BLASTN version 2.2.29 [28] Sequence homology was compared with $16 \mathrm{~S}$ rRNA gene sequences available in the database using the FASTA algorithm. The 16S rRNA gene sequences of other Streptomyces, representing the type strains of Streptomyces species, were retrieved from the GenBank. The sequence was aligned using Clustal W ver. 2.01, and the phylogenetic tree was constructed using MEGA ver. 6 by the neighbor-joining method (Figure S1). The assigned GenBank DNA sequences DA-1, DA-2, DA-3, DA4, KM-1, KM-2, KM-3, KM-5, KM-6, KM-8, SA1, SA2, SA3, SA4, SA4, SA5, SA6, SA7, and SA8. The accession numbers are mentioned in Table 1 , and phylogenetic analysis revealed that 18 isolates shared some evolutionary similarities (Supplementary Materials, Figure S1).

3.5. Genome-Wide Analysis of $16 S$ rRNA. The multiple copies of $16 \mathrm{~S}$ rRNA are found in 27 Streptomyces species in their whole-genome sequences. In 7 species, DNA sequences in multiple copies of $16 \mathrm{~S}$ rRNA are highly conserved, but in 20 others, there is little variation and per-site gaps in the aligned predicted by multiple sequences. Streptomyces violaceusniger Tu 4113 has only one 16S rRNA in the genome. The numerous copy of 16S rRNA present in the assembled genome sequencing of Streptomyces is given in Supplementary Materials, Table S3. Our analysis revealed that DNA sequencing of a single copy of $16 \mathrm{~S}$ rRNA in the genome could be sufficient for the identification of genus Streptomyces from class actinomycetes. 

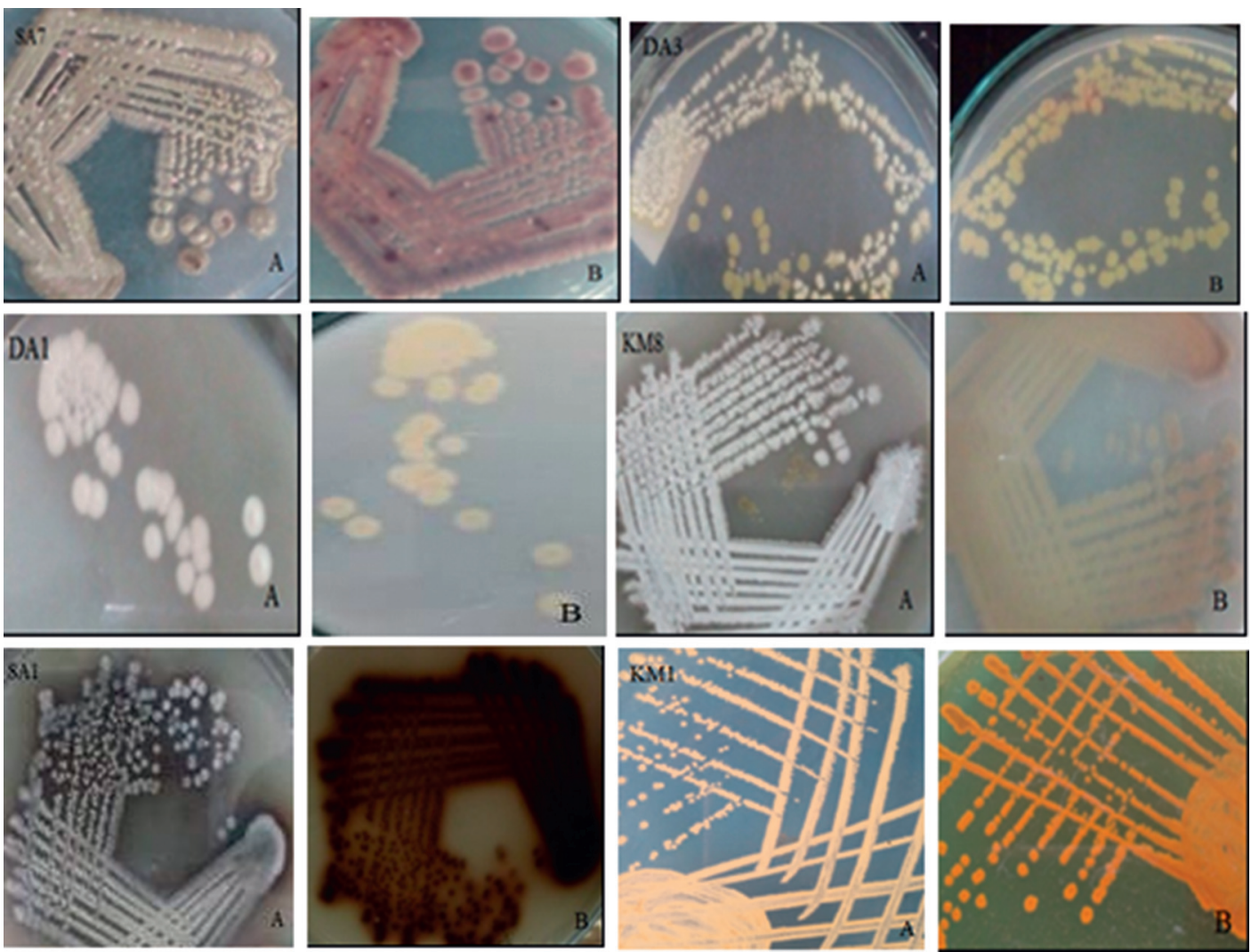

FIgURE 1: Colony morphology indicating aerial (a) and substrate (b) mycelia of isolated Streptomyces species.

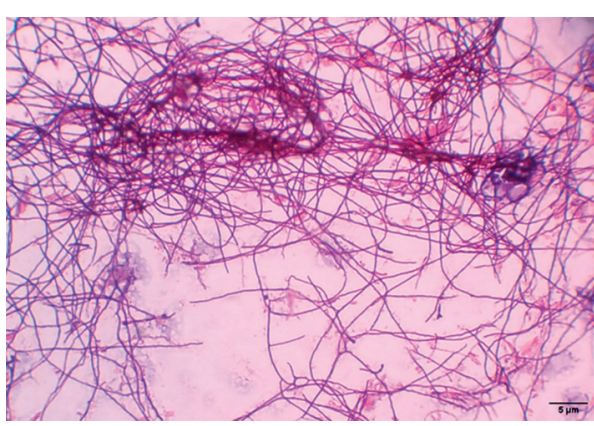

(a)

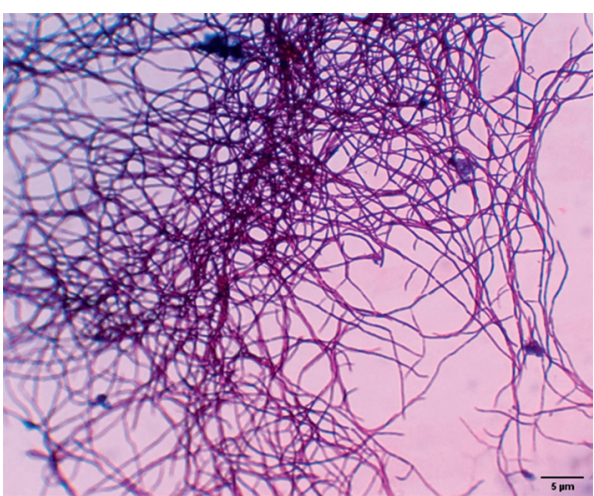

(c)

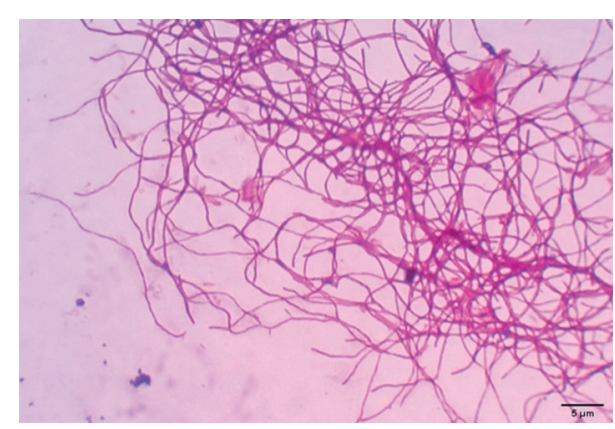

(b)

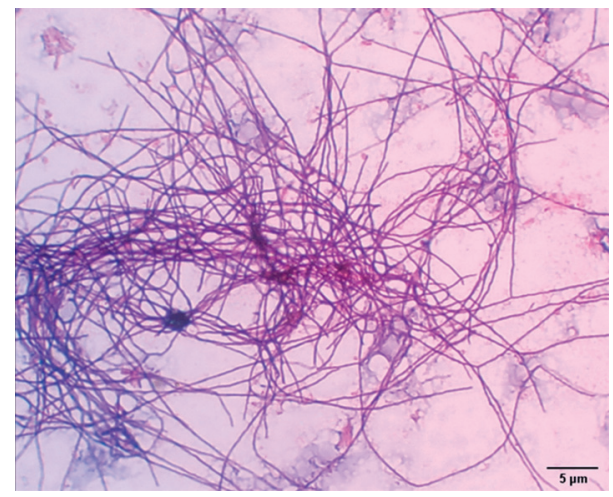

(d)

FIGURE 2: Gram staining of isolated actinomycetes under 100x magnification. 


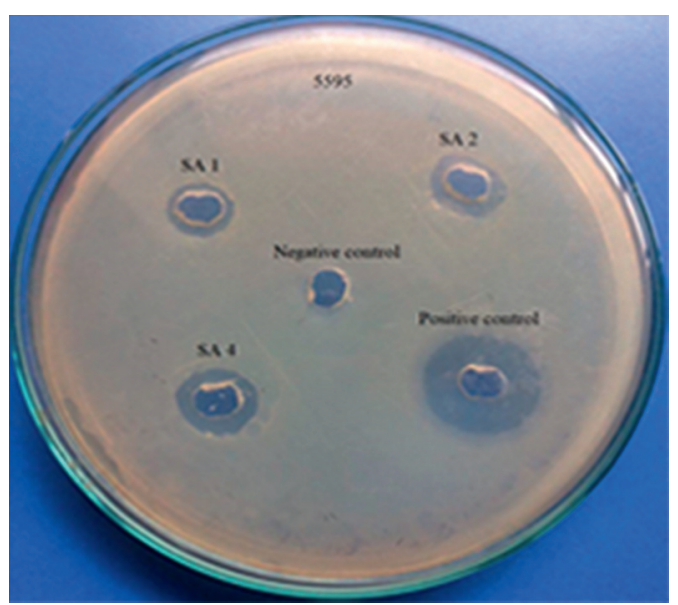

(a)

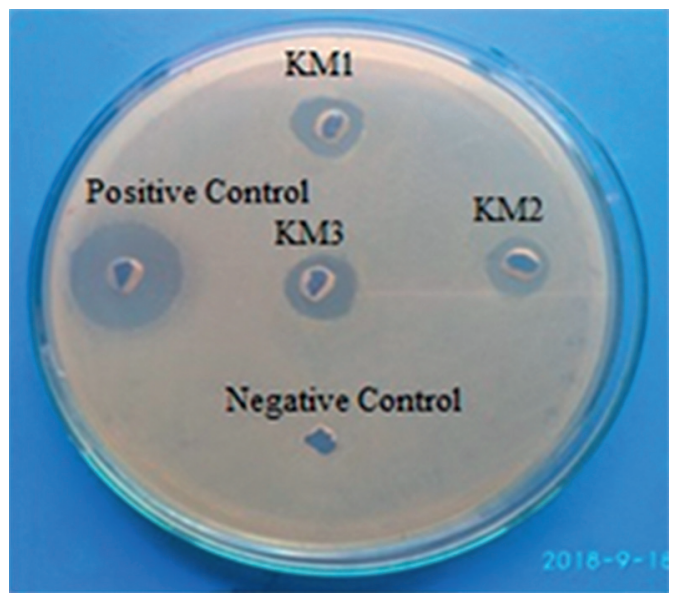

(c)

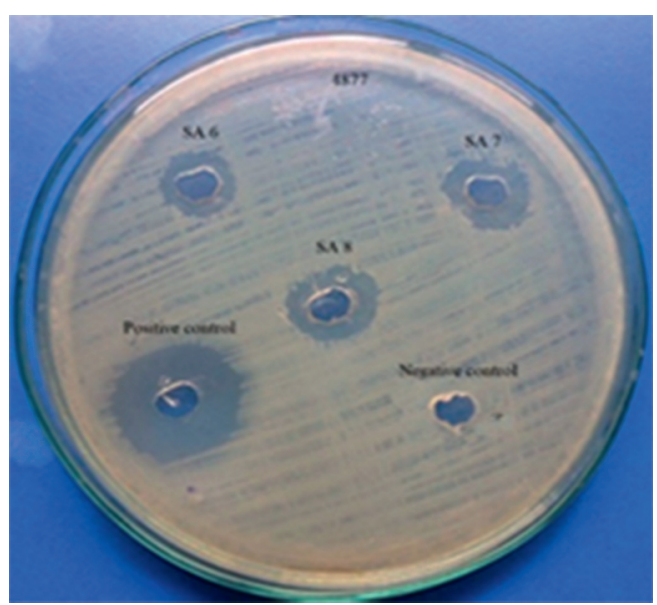

(b)

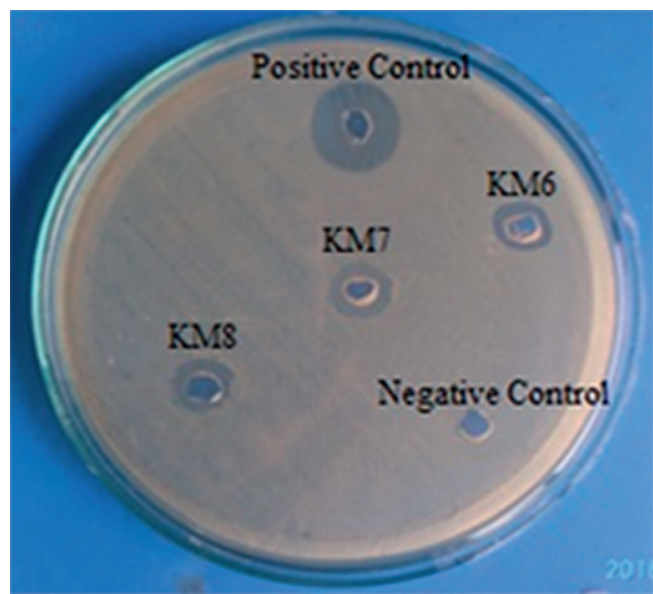

(d)

FIGURE 3: Antibacterial activity against ESBL-producing E. coli shown by crude extract of Streptomyces species.

\section{Discussion}

The present study outlines molecular phylogeny, and antimicrobial activity of Streptomyces isolated from Nepalese soil. Till now, more than 7,000 compounds produced by Streptomyces species have been identified [6]. Continuous screening of Streptomyces will lead to the discovery of numbers of new compounds with diverse applications [29]. Thus, the reported 18 Streptomyces species from different environmental niches of Nepal would be beneficial for future antibiotics discovery program.

In this study, the ethyl acetate extracts showed antimicrobial activity against ESBL-producing E. coli. Previously, Ramachandran et al. have also suggested that ethyl acetate extract of actinomycetes has antibacterial activity against ESBL-producing E. coli [30], and this solvent is considered better for the extraction of active metabolites from actinomycetes [31]. In several reports, secondary metabolites from actinomycetes have shown the higher inhibition against Gram-negative bacteria; among them, E. coli is more susceptible. However, due to the presence of double-membrane barrier and transmembrane efflux, Gram-negative bacteria are more resistant to antimicrobial compounds as compared to Gram-positive bacteria [32]. Hence, actinomycetes that produce secondary metabolites against Gram-negative might play a significant role against antibiotic-resistant bacteria, such as ESBL-producing E. coli. Previous studies suggested that ethyl acetate extract of actinomycetes, isolated from Nepalese soil of different altitudes, can produce secondary metabolites, which showed antimicrobial activity against different ATCC strains [33-36].

To our knowledge, the antimicrobial activity against ESBL-producing E. coli using secondary metabolites of Streptomyces and their molecular characterization have not been reported earlier from Nepal.

From the analysis of partial $16 \mathrm{~S}$ rRNA sequences deposited in GenBank (Table 1), it was revealed that all isolates belong to a species of the genus Streptomyces with 80-99\% similarity with the $16 \mathrm{~S}$ rRNA sequence of its closely related species. Bacterial genetic marker, $16 \mathrm{~S}$ rRNA, often exists as a multigene family or operons in chromosomal DNA. The function of this gene over time has not been changed, and the size of this gene is large enough for the informatics purposes [37]. The complete gene sequence of $16 \mathrm{~S}$ rRNA consists of approximately $1,500 \mathrm{bp}$ with significantly conserved regions and nine variable regions that allow broad 
TABLE 1: 16S rRNA sequence analysis of Streptomyces species and potent secondary metabolites.

\begin{tabular}{|c|c|c|c|c|}
\hline \multirow{2}{*}{ Sample no. } & \multirow{2}{*}{ Accession number } & \multicolumn{3}{|l|}{ Match in the databases } \\
\hline & & Species & Identity (\%) & Identifier \\
\hline \multirow{2}{*}{ SA1 } & \multirow{2}{*}{ LC427859 } & Streptomyces spp. ZG731 & 96 & GQ985455.1 \\
\hline & & Streptomyces coelicoflavus strain HQA809 & 93 & KT758401.2 \\
\hline \multirow{2}{*}{ SA2 } & \multirow{2}{*}{ LC425654 } & Streptomyces spp. BCG69 & 80 & KF956734.1 \\
\hline & & Streptomyces spp. 219839 & 80 & HQ992728.1 \\
\hline \multirow{2}{*}{ SA3 } & \multirow{2}{*}{ LC427860 } & Streptomyces spp. strain D3 & 97 & KX762051.1 \\
\hline & & Streptomyces lividans strain $\mathrm{jx}-02$ & 97 & KC898819.1 \\
\hline \multirow{2}{*}{ SA4 } & \multirow{2}{*}{ LC427861 } & Streptomyces spp. strain D3 & 98 & KX762051.1 \\
\hline & & Streptomyces lividans strain YLA0 & 98 & KT362142.1 \\
\hline \multirow{2}{*}{ SA5 } & \multirow{2}{*}{ LC427862 } & Streptomyces spp. CMU-AC2 & 96 & LC073311.1 \\
\hline & & Streptomyces spp. CMU-AB225 & 96 & LC073310.1 \\
\hline \multirow{2}{*}{ SA6 } & \multirow{2}{*}{ LC427863 } & Streptomyces spp. JSM 147611 & 96 & KR817740.1 \\
\hline & & Streptomyces violascens strain G8A-22 & 96 & HQ238389.1 \\
\hline \multirow{2}{*}{ SA7 } & \multirow{2}{*}{ LC427864 } & Streptomyces spp. DHS C014 & 97 & KP986577.1 \\
\hline & & Streptomyces tumenensis & 97 & AM180560.1 \\
\hline \multirow{2}{*}{ SA8 } & \multirow{2}{*}{ LC427865 } & Uncultured Streptomyces spp. clone T1S-05 & 82 & GQ369231.1 \\
\hline & & Streptomyces ryensis strain zw24 & 83 & MH337934.1 \\
\hline \multirow{2}{*}{ KM1 } & \multirow{2}{*}{ MT463712 } & Streptomyces spp. 34005 & 98 & GU263848.1 \\
\hline & & Streptomyces strain P14S1 & 98 & KX673842.1 \\
\hline \multirow{2}{*}{ KM2 } & \multirow{2}{*}{ MT463715 } & Streptomyces globisporus subsp. globisporus isolate XSD-114 & 85.1 & EU273549.1 \\
\hline & & Streptomyces cavourensis strain TSM 11 & 85 & MK789724.1 \\
\hline \multirow{2}{*}{ KM3 } & \multirow{2}{*}{ MT463732 } & Streptomyces pratensis strain EA5 & 93.2 & KU973961.1 \\
\hline & & Streptomyces spp. QLS12 & 93.5 & KU973961.1 \\
\hline \multirow{2}{*}{ KM5 } & \multirow{2}{*}{ MT464457 } & Streptomyces coelicoflavus strain 3-2 & 98.3 & KJ571034.1 \\
\hline & & Streptomyces coelicoflavus strain HQA020 & 98.1 & KT758352.1 \\
\hline \multirow{2}{*}{ KM6 } & & Streptomyces spp. 193322 & 96.2 & KU982617.1 \\
\hline & MT464460 & Streptomyces parvus strain T23 & 96.2 & KU317906.1 \\
\hline KM8 & & Streptomyces canus strain IMCC 34906 & 97.4 & MK138629.1 \\
\hline KM8 & МТ464462 & Streptomyces spp. strain ST228 & 97.4 & KX906839.1 \\
\hline & & Streptomyces globisporus strain AHS10 & 99 & KU981096.1 \\
\hline DA1 & MT441544 & Streptomyces violascens strain EA27 & 98.2 & KU973982.1 \\
\hline $\mathrm{DA?}$ & MT450245 & Streptomyces naganishii NRRLB-1816 & 98.4 & NR_043831.1 \\
\hline DA2 & MT459245 & Streptomyces spp. FXJ1.447 & 98.4 & KP126357.1 \\
\hline & & Streptomyces pratensis 190525 & 97.1 & KU973967.1 \\
\hline DA3 & MT459275 & Streptomyces spp. strain WA22-1-2 & 97 & KY206810.1 \\
\hline & & Streptomyces spp. strain HN25 & 98.7 & MF397913.1 \\
\hline DA4 & MT463685 & Streptomyces diastaticus subsp. ardesiacus strain AR-39 & 98.7 & KX055839.1 \\
\hline
\end{tabular}

taxonomic spectrum and taxonomic discrimination, respectively. However, there is some discrepancy regarding the molecular identification of bacterial species based on $16 \mathrm{~S}$ rRNA. Guo et al. have claimed that sequencing of $16 \mathrm{~S}$ rRNA is not useful for closely related strains and only limited to the distinction of slightly related Streptomycetes [38].

Some studies have reported that multiple copies of $16 \mathrm{~S}$ rRNA are limited and have less probability of affecting the phylogenetic analysis of the species because, in most cases, the sequence of these multiple copies is entirely or nearly identical [39-41]. However, others have shown that with an increase in the number of multiple copies of $16 \mathrm{~S}$ rRNA, the variation in sequence also increases [42], and the 16S rRNA sequence is not enough criteria to assure species identity [43].

In silico analysis of $16 \mathrm{~S}$ rRNA of 174 actinobacteria showed the phylum contains $3.402 \pm 1.720$ average copies of 16S rRNA, of which $1.569 \pm 0.869$ copies are not $100 \%$ similar to other copies. The results infer that the average maximum variation is $1.121 \pm 2.400$, along with an average minimum similarity of $99.927 \pm 0.158$ based on multiple copies of 16S rRNA analysis [44]. Ten Streptomyces strains isolated from three different lichens were found to have similar 16S rRNA gene sequences [45, 46]. The analysis of multiple copies of 16S rRNA in different Nocardia strains suggests that only BLAST analysis could not confirm the species present in the isolates [47]. Two copies of $16 \mathrm{~S}$ rRNA genes contained in actinomycete, Thermobispora bispora, differ in nucleotides sequence at 98 positions $(6.4 \%$ of complete nucleotides sequence) having six regions of insertion and deletions [48]. A study suggested transitional and transversional substitutions as two distinct mechanisms for the occurrence of multiple copies of 16S rRNA in 33 Streptomyces strains (6.9\%) out of 475 isolates [49]. Thus, the sequencing of multiple copies of $16 \mathrm{~S}$ rRNA seems to be not useful for the identification of species.

\section{Conclusion}

The present study demonstrates that Streptomyces species isolated from different altitudes of Nepal showed potential 
antibacterial activity against ESBL-producing E. coli. This report demands further study on Streptomyces spp. of Nepal to uncover novel secondary metabolites, which could be an ideal option for the treatment of infections caused by drugresistant pathogens. We also suggest that the molecular sequencing of multiple copies of the $16 \mathrm{~S}$ rRNA is not necessary for species identification of Streptomycetes, rather fatty acid methyl ester (FAME) analysis, profiling of metabolites, and DNA-DNA hybridization are required for this purpose.

\section{Data Availability}

DNA accession numbers are available on GenBank.

\section{Conflicts of Interest}

The authors declare that they have no conflicts of interest regarding the publication of this paper.

\section{Authors' Contributions}

Dawa Dindu Sherpa, Krishna Prakash Malla, and Sunil Shrestha contributed equally to this work. NP designed the research; DDS, KPM, SS, and KK carried out experiments; NP, BPM, SK, BR, NR, and BRB analyzed the data; and NP wrote the manuscript.

\section{Acknowledgments}

The authors would like to thank Mr. Raju Shreastha for providing ESBL-producing E. coli samples. The authors are also thankful to the University Grants Commission, Nepal. This study was financially supported under the Collaborative Research Grants (Award no. CRG-75/76-S\&T-1) by the University Grants Commission, Nepal, to Niranjan Parajuli.

\section{Supplementary Materials}

Supplementary Figure S1. Neighbor-joining phylogenetic tree of $16 \mathrm{~S}$ rRNA from 18 isolates. Horizontal branch lengths proportional to the estimated number of nucleotide substitutions, and bootstrap probabilities (as percentages), as determined for 1000 resamplings, are given above or beside the internal branches. The bar in the lower-left corner indicates 0.2 amino acid substitutions per amino acid for the branch length. Supplementary Table S1. Cultural features of Streptomyces species isolated from different parts of Nepal. Supplementary Table S2. Antimicrobial activity of some Streptomyces species against ESBL-producing E. coli. Supplementary Table S3. Multiple copy numbers of 16S rRNA present in the assembled genome sequencing of Streptomyces given in the website (JGI IMG Integrated Microbial Genomes and Microbiomes, 2019). (Supplementary Materials)

\section{References}

[1] O. Genilloud, "Actinomycetes: still a source of novel antibiotics," Natural Product Reports, vol. 34, no. 10, pp. 1203-1232, 2017.
[2] J. Hug, C. Bader, K. Cirnski, and R. Müller, "Concepts and methods to access novel antibiotics from actinomycetes," Antibiotics, vol. 7, no. 2, p. 44, 2018.

[3] K. Poole, "Mechanisms of bacterial biocide and antibiotic resistance," Journal of Applied Microbiology, vol. 92, no. s1, pp. 55S-64S, 2002.

[4] D. J. Payne, M. N. Gwynn, D. J. Holmes, and D. L. Pompliano, "Drugs for bad bugs: confronting the challenges of antibacterial discovery," Nature Reviews Drug Discovery, vol. 6, no. 1, pp. 29-40, 2007.

[5] C. Olano, C. Méndez, and J. Salas, "Antitumor compounds from marine actinomycetes," Marine Drugs, vol. 7, no. 2, pp. $210-248,2009$.

[6] J. Bérdy, "Bioactive microbial metabolites," The Journal of Antibiotics, vol. 58, no. 1, pp. 1-26, 2005.

[7] K. F. Chater, “"Morphological and physiological differentiation in Streptomyces," cold spring," Harbor Monograph Archive, vol. 16, pp. 89-115, 1984.

[8] D. A. Hopwood, "Highlights of Streptomyces genetics," Heredity, vol. 123, no. 1, pp. 23-32, 2019.

[9] M. Watve, R. Tickoo, M. Jog, and B. Bhole, "How many antibiotics are produced by the genus Streptomyces?" Archives of Microbiology, vol. 176, no. 5, pp. 386-390, 2001.

[10] S. A. Waksman, A. Schatz, and D. M. Reynolds, "Production of antibiotic substances by actinomycetes," Annals of the New York Academy of Sciences, vol. 1213, no. 1, pp. 112-124, 2010.

[11] J. Bérdy, "Thoughts and facts about antibiotics: where we are now and where we are heading," The Journal of Antibiotics, vol. 65, no. 8, pp. 385-395, 2012.

[12] P. Sivalingam, K. Hong, J. Pote, and K. Prabakar, "Extreme environment Streptomyces: potential sources for new antibacterial and anticancer drug leads?" International Journal of Microbiology, vol. 2019, pp. 1-20, 2019.

[13] M. Harir, H. Bendif, M. Bellahcene, Z. Fortas, and R. Pogni, "Streptomyces secondary metabolites," Basic Biology and Applications of Actinobacteria, vol. 6, pp. 99-122, 2018.

[14] T. D. Gurung, C. Sherpa, V. P. Agrawal, and B. Lekhak, "Isolation and characterization of antibacterial actinomycetes from soil samples of kalapatthar, mount everest region," Nepal Journal of Science and Technology, vol. 10, pp. 173-182, 1970.

[15] P. S. Kumar, J. P. P. Raj, V. Duraipandiyan, and S. Ignacimuthu, "Antibacterial activity of some actinomycetes from Tamil Nadu, India," Asian Pacific Journal of Tropical Biomedicine, vol. 2, no. 12, pp. 936-943, 2012.

[16] C. K. Okoro, R. Brown, A. L. Jones et al., "Diversity of culturable actinomycetes in hyper-arid soils of the Atacama Desert, Chile," Antonie Van Leeuwenhoek, vol. 95, no. 2, pp. 121-133, 2009.

[17] M. E. Rateb, W. E. Houssen, W. T. A. Harrison et al., "Diverse metabolic profiles of a Streptomyces strain isolated from a hyper-arid environment," Journal of Natural Products, vol. 74, p. $1965,2011$.

[18] P.-L. Lu, Y.-C. Liu, H.-S. Toh et al., "Epidemiology and antimicrobial susceptibility profiles of Gram-negative bacteria causing urinary tract infections in the Asia-Pacific region: 2009-2010 results from the Study for Monitoring Antimicrobial Resistance Trends (SMART)," International Journal of Antimicrobial Agents, vol. 40, pp. S37-S43, 2012.

[19] I. Saadoun, K. M. Hameed, and A. Moussauui, "Characterization and analysis of antibiotic activity of some aquatic actinomycetes," Microbios, vol. 99, no. 394, pp. 173-179, 1999.

[20] M. K. Kharel, M. D. Shepherd, S. E. Nybo et al., "Isolation of Streptomyces species from soil," Current Protocols in Microbiology, vol. 19, 2010. 
[21] W. Whitman, M. Goodfellow, P. Kämpfer et al., Bergey's Manual of Systematic Bacteriology: Volume 5: The Actinobacteria, Springer-Verlag, New York, NY, USA, 2nd edition, 2012.

[22] J. Sambrook and D. W. Russell, "Molecular cloning: a laboratory manual," Cold Spring Harbor Laboratory Press, vol. 1, p. 112, 2001.

[23] P. Jose and S. R. Jebakumar, "Phylogenetic diversity of actinomycetes cultured from coastal multipond solar saltern in Tuticorin, India," Aquatic Biosystems, vol. 8, no. 1, p. 23, 2012.

[24] M. Benhadj, D. Gacemi-Kirane, T. Menasria, K. Guebla, and Z. Ahmane, "Screening of rare actinomycetes isolated from natural wetland ecosystem (Fetzara Lake, northeastern Algeria) for hydrolytic enzymes and antimicrobial activities," Journal of King Saud University-Science, vol. 31, no. 4, pp. 706-712, 2019.

[25] N. A. Al-Dhabi, G. A. Esmail, V. Duraipandiyan, M. Valan Arasu, and M. M. Salem-Bekhit, "Isolation, identification and screening of antimicrobial thermophilic Streptomyces sp. AlDhabi-1 isolated from Tharban hot spring, Saudi Arabia," Extremophiles, vol. 20, no. 1, pp. 79-90, 2016.

[26] R. Shrestha, S. Khanal, P. Poudel et al., "Extended spectrum $\beta$-lactamase producing uropathogenic Escherichia coli and the correlation of biofilm with antibiotics resistance in Nepal," Annals of Clinical Microbiology Antimicrobials, vol. 18, p. 42, 2019.

[27] O. Mustafa, A. U. Tamer, and A. Cem, "Antibacterial activity of some actinomycetes isolated from farming soils of Turkey," African Journal of Biotechnology, vol. 3, no. 9, pp. 441-446, 2004.

[28] A. Morgulis, G. Coulouris, Y. Raytselis, T. L. Madden, R. Agarwala, and R. Schäffer, "Database indexing for production MegaBLAST searches," Bioinformatics, vol. 24, no. 24, pp. 1757-1764, 2008.

[29] S. T. Williams, M. Goodfellow, G. Alderson, E. M. H. Wellington, P. H. A. Sneath, and M. J. Sackin, "Numerical classification of Streptomyces and related genera," Microbiology, vol. 129, no. 6, pp. 1743-1813, 1983.

[30] G. Ramachandran, G. Rajivgandhi, M. Maruthupandy, and N. Manoharan, "Isolation and identification of antibacterial compound from marine endophytic actinomycetes against multi drug resistant bacteria," Annals of Microbiology and Immunology, vol. 1, p. 1003, 2018.

[31] A. Gorajana, S. Vinjamuri, B. V. V. S. N. Kurada et al., "Resistoflavine, cytotoxic compound from a marine actinomycete, Streptomyces chibaensis AUBN1/7," Microbiological Research, vol. 162, no. 4, pp. 322-327, 2007.

[32] H. I. Zgurskaya and H. Nikaido, "Multidrug resistance mechanisms: drug efflux across two membranes," Molecular Microbiology, vol. 37, no. 2, pp. 219-225, 2000.

[33] A. Sapkota, A. Thapa, A. Budhathoki, M. Sainju, P. Shrestha, and S. Aryal, "Isolation, characterization, and screening of antimicrobial-producing actinomycetes from soil samples," International Journal of Microbiology, vol. 2020, 2020.

[34] T. D. Gurung, C. Sherpa, V. P. Agrawal, and B. Lekhak, "Isolation and characterization of antibacterial actinomycetes from soil samples of Kalapatthar, Mount Everest Region," Nepal Journal of Science and Technology, vol. 10, pp. 173-182, 2009.

[35] K. Rai, S. Khadka, and B. Shrestha, "Actinomycetes: isolation, characterization and screening for antimicrobial activity from different sites of Chitwan, Nepal," International Journal of Microbiology and Biotechnology, vol. 3, no. 1, p. 25, 2018.
[36] B. Pandey, P. Ghimire, and V. P. Agrawal, "Studies on the antibacterial activity of the actinomycetes isolated from the khumbu region of Nepal," International Journal of Microbiology and Biotechnology, vol. 23, pp. 44-53, 2004.

[37] J. Patel, "16S rRNA gene sequencing for bacterial pathogen identification in the clinical laboratory," Molecular Diagnosis, vol. 6, no. 4, pp. 313-321, 2001.

[38] Y. Guo, W. Zheng, X. Rong, and Y. Huang, "A multilocus phylogeny of the Streptomyces griseus $16 \mathrm{~S}$ rRNA gene clade: use of multilocus sequence analysis for streptomycete systematics," International Journal of Systematic and Evolutionary Microbiology, vol. 58, no. 1, pp. 149-159, 2008.

[39] T. P. Tourova, "Copy number of ribosomal operons in prokaryotes and its effect on phylogenetic analyses," Microbiology, vol. 72, no. 4, pp. 389-402, 2003.

[40] T. Coenye and P. Vandamme, "Intragenomic heterogeneity between multiple $16 \mathrm{~S}$ ribosomal RNA operons in sequenced bacterial genomes," FEMS Microbiology Letters, vol. 228, no. 1, pp. 45-49, 2003.

[41] N. Engene and W. H. Gerwick, "Intra-genomic 16S rRNA gene heterogeneity in cyanobacterial genomes," Fottea, vol. 11, no. 1, pp. 17-24, 2011.

[42] T. Větrovský and P. Baldrian, "The variability of the 16S rRNA gene in bacterial genomes and its consequences for bacterial community analyses," PLoS One, vol. 8, 2013.

[43] G. E. Fox, J. D. Wisotzkey, and P. Jurtshuk, "How close is close: $16 \mathrm{~S}$ rRNA sequence identity may not Be sufficient to guarantee species identity," International Journal of Systematic Bacteriology, vol. 42, no. 1, pp. 166-170, 1992.

[44] J. C. Ibal, H. Q. Pham, C. E. Park, and J. H. Shin, "Information about variations in multiple copies of bacterial 16S rRNA genes may aid in species identification," PLoS One, vol. 14, 2019.

[45] D. Parrot, S. Antony-Babu, L. Intertaglia et al., "Littoral lichens as a novel source of potentially bioactive Actinobacteria," Scientific Reports, vol. 5, 2015.

[46] S. Antony-Babu, D. Stien, V. Eparvier et al., "Multiple Streptomyces species with distinct secondary metabolomes have identical 16S rRNA gene sequences," Scientific Reports, vol. 7, 2017.

[47] P. S. Conville and F. G. Witebsky, "Analysis of multiple differing copies of the 16S rRNA gene in five clinical isolates and three type strains of Nocardia species and implications for species assignment," Journal of Clinical Microbiology, vol. 45, no. 4, pp. 1146-1151, 2007.

[48] Y. Wang, Z. Zhang, and N. Ramanan, "The actinomycete Thermobispora bispora contains two distinct types of transcriptionally active $16 \mathrm{~S}$ rRNA genes," Journal of Bacteriology, vol. 179, no. 10, pp. 3270-3276, 1997.

[49] K. Ueda, T. Seki, T. Kudo, T. Yoshida, and M. Kataoka, "Two distinct mechanisms cause heterogeneity of 16S rRNA," Journal of Bacteriology, vol. 181, no. 1, pp. 78-82, 1999. 